\title{
Electromagnetic Matched-Field Processing: Basic Concepts and Tropospheric Simulations
}

\author{
Donald F. Gingras, Member, IEEE, Peter Gerstoft, and Neil L. Gerr
}

\begin{abstract}
Recent waveguide array processing methods have incorporated the physics of wave propagation as an integral part of the processing. Matched-field processing (MFP) refers to signal and array processing techniques in which, rather than a planewave arrival model, complex-valued (amplitude and phase) field predictions for propagating signals are used. Matchedfield processing has been successfully applied in ocean acoustics. In this paper, the extension of MFP to the electromagnetic domain, i.e., electromagnetic (EM) MFP (EM-MFP) is described. Simulations of EM-MFP in the tropospheric setting suggest that under suitable conditions, EM-MFP methods can enable EM sources to be both detected/localized and used as sources of opportunity for estimating the environmental parameters that determine EM propagation.
\end{abstract}

Index Terms-Signal processing antennas.

\section{INTRODUCTION}

$\mathbf{R}$ ECENT research on array processing for ocean acoustics has included a significant effort to use precise full wave propagation models and efficient parameter search algorithms in order to jointly estimate both signal source-location and environmental parameters. These methods are referred to as matched-field processing (MFP) because they exploit the fullfield structure of signals propagating in the waveguide. MFP is a generalization of plane wave beamforming wherein the "steering or replica" vector is derived from a solution of the wave equation for a point source. Because the plane wave model is not generally an appropriate model for signals propagating in a waveguide, MFP provides improved localization performance. Excellent overviews of MFP methods and their application to ocean acoustics are given in [1] and [2].

Research on MFP initially focused on problems of signal source detection and localization, given assumed known environmental parameters. Unfortunately, due to limitations in ocean modeling and measurement capabilities and the effects of stochastic small-scale ocean phenomena such as internal waves, the environmental parameters and, hence, the acoustic predictions inherent to acoustic MFP were often subject to error. Recognition of this problem motivated a good deal of recent MFP research. For example, in [3]-[6] the deterministic sensitivity of MFP to misspecification of environmental

Manuscript received June 21, 1996; revised January 20, 1997.

D. F. Gingras is with the Naval Command, Control and Ocean Surveillance Center, San Diego, CA, 92152 USA.

P. Gerstoft is with the Marine Physics Laboratory, University of California at San Diego, San Diego, CA 92037 USA.

N. L. Gerr is with the Surveillance, Communications and Electronic Combat Division, Office of Naval Research, Arlington, VA 22217 USA.

Publisher Item Identifier S 0018-926X(97)07224-4. parameters was examined and MFP techniques robust to such misspecification were described. The use of a probabilistic framework in which the environmental parameters were specified with uncertainty or as random variables also yielded robust estimation methods [7]-[9]. An alternative approach termed "focalization" whereby the environmental parameters were estimated jointly with source-location parameters was introduced in [10]. Experimental verification that the mismatch problem could be overcome by joint estimation of both source and environmental parameters was reported in [11].

MFP methods for joint source-localization and environmental parameter estimation have not previously been applied in the electromagnetic domain. In this paper, the extension of MFP to the electromagnetic domain is described, i.e., electromagnetic MFP (EM-MFP), as a means for simultaneously localizing an EM source and estimating the environmental parameters associated with the propagation of the source signal. While methods for estimating environmental parameters using EM field measurements have been successful and there are a number of papers on this subject [12]-[15], in this previous work, the source-receiver geometry was known and, in fact, was controlled so as to optimize information about environmental parameters. In Section II, the basic concepts of EM-MFP are formalized and the RF propagation issues associated with tropospheric propagation in the very-highfrequency (VHF) to extremely high-frequency (EHF) bands are introduced. In Section III, the potential performance of EM-MFP for a tropospheric application is illustrated using synthetic data. The results suggest that under suitable conditions, EM-MFP methods can enable RF emitters to be simultaneously localized and used as sources of opportunity for estimating environmental parameters thereby obviating the need for a large instrumented campaign. In Section IV, the basic findings are summarized and possible directions for future research are described.

\section{BASIC CONCEPTS OF MFP}

Early work in acoustic MFP typically treated cases in which it was assumed that all parameters except for two (e.g., source range and depth for MFP using a vertical array) were known without error. Solution then involved computing the so-called range-depth "ambiguity function," a two-dimensional (2-D) surface whose maximum, generally found by visual inspection or exhaustive search, formed the source-location estimate. In what follows, such a case in the setting of EM-MFP is considered along with a more complicated case in which the environmental parameters that determine propagation are also 
unknown. Solution in the latter case requires sophisticated search techniques to find the global maximum in a space of dimension greater than two.

It is helpful to formulate MFP as an inverse problem in that the observations are known to be related to an unknown parameter vector through a known nonlinear function. For MFP the observations are the antenna/hydrophone array data samples, the nonlinear function is the differential equation governing wave propagation, and the parameter vector is comprised of the source-location coordinates and the environmental parameters. The goal is to estimate the parameter vector from the observations using knowledge about the nonlinear function.

Inverse problems such as that posed by MFP may be solved using nonlinear optimization and a forward model. One first constrains the solution set to a bounded range of possible values for each parameter, the so-called "feasible region." An objective function which provides a measure of similarity between the observed array signal samples and those predicted based on the forward-propagation model is optimized over a discretized version of the feasible region. In most situations, the set contains a great number of "feasible solutions" and optimization via exhaustive search is not viable. Furthermore, the objective function may contain many local maxima (sidelobes) precluding the use of gradient descent methods. Efficient global-optimization methods such as simulated annealing (SA) and genetic algorithms (GA) have been successfully employed [10], [16] in acoustic MFP.

Thus, whether applied to acoustics or electromagnetics, MFP requires the following basic ingredients: 1) spatial samples of the signal field from an array of sensors; 2) full-wave forward propagation model and a priori description of the environmental parameters; 3) objective function that relates the array data to the propagation model and environmental parameters; and 4) efficient algorithm for searching a high-dimensional parameter space coupled with a posteriori analysis of the results. These basic ingredients will be described more fully in the sections that follow. Throughout the parameter vector denoted by $\mathrm{m}$ will, in the most general case, contain the bounded set of feasible values for signal source- and receiver-location coordinates and environmental parameters.

\section{A. Array Data for MFP}

MFP in ocean acoustics typically employs a vertical array of hydrophones of sufficient length to "adequately" sample the acoustic field over some portion of the water column. For example, in [11], a vertical array containing 48 hydrophones spanning 11 wavelengths at $170 \mathrm{~Hz}$ was used as the basis for inversion of acoustic field data. Vertical antenna arrays have also been employed in the electromagnetic domain. Webster [17] used a vertical array containing 16 antenna elements spanning 666 wavelengths at $16.65 \mathrm{GHz}$ to estimate the timevarying multipath arrival structure (multipath amplitude and angles-of-arrival). Webster's results indicate that: 1) the angleof-arrival structure was rich in that there were always at least five different path arrivals and 2) the angle-of-arrival structure was time varying. The first observation strongly suggests that there is information in the arrival structure that

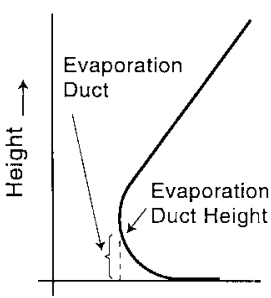

(a)

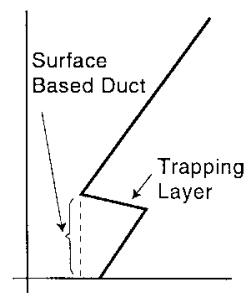

Modified Refractivity M

(b)

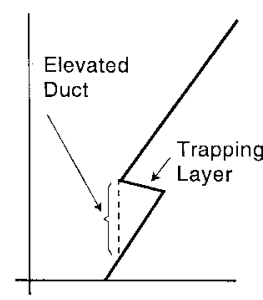

(c)
Fig. 1. Modified refractivity $M$ versus height. (a) Evaporation duct. (b) Surface duct. (c) Elevated duct.

can be exploited through EM-MFP for the purpose of sourcelocation and environmental-parameter estimation. The second observation implies that for EM-MFP it will not be sufficient to use a priori environmental parameters for source-location estimation. Rather, EM-MFP is likely to require the joint estimation of these parameters. Furthermore, for EM-MFP to be of utility in an operational setting, the estimation will have to be conducted on a time scale consistent with the time-varying nature of the EM channel.

\section{B. Propagation Modeling}

The mechanisms that govern the propagation of radio waves within the troposphere can be separated into two classes-standard and nonstandard. Standard propagation mechanisms are associated with a refractive index that decreases monotonically with height, generally in a linear fashion at low altitudes but exponentially at higher elevations. Nonstandard propagation mechanisms are associated with vertical distributions of the refractive index that are not monotonically decreasing and so create trapping layers and ducting.

The refractive index $n(\mathbf{r}, t)$, where the spatial variables range $x$ and height $z$ are denoted by the vector $\mathbf{r}$, is defined as the ratio of the velocity of propagation of an EM wave in a vacuum to that in the medium and the refractive index $N(\mathrm{r}, t)$ is defined as $N=(n-1) 10^{6}$. Refractivity in the atmosphere is known to be a function of atmospheric pressure, air temperature, and humidity. In a "standard" well-mixed atmosphere, both temperature and humidity decrease with increasing elevation such that $N(\mathbf{r}, t)$ decreases at a rate of about $39 N$ units per kilometer. A more convenient method for quantifying refractivity as it relates to the occurrence of ducting is with the use of the modified refractivity $M(\mathrm{r}, t)$ defined in terms of $N(\mathrm{r}, t)$ as

$$
M=N+(h / a) \times 10^{6}
$$

where $h$ is height above the earth's surface and $a$ is the earth's radius. $M$ is useful for identifying trapping layers since trapping occurs for all negative $M$ gradients.

Generally speaking, there are three types of ducts, these being (in order of increasing elevation) the evaporation duct, surface ducts, and elevated ducts, as depicted in Fig. 1. In what follows we will give a brief description of these ducts and associated phenomena. For additional information see Hitney [18]. 
In the marine environment, the evaporation duct is a nearly ever-present propagation mechanism created by the rapid decrease of moisture immediately above the ocean surface. For continuity reasons, the air adjacent to the ocean is essentially fully saturated with water vapor. This high relative humidity decreases rapidly with increasing height in the first few meters until an ambient value is reached. The rapid decrease in humidity creates a trapping layer adjacent to the ocean surface, as shown in Fig. 1(a). The height of the evaporation duct varies between about 0 and $40 \mathrm{~m}$. The frequencies that an evaporation duct can support are dependent on the duct height with a lower limit of about $3 \mathrm{GHz}$.

Surface ducts, as illustrated by Fig. 1(b), are usually created by a trapping layer that occurs up to several hundred meters in height, although they can be created by a trapping layer adjacent to the surface. The condition for a surface duct to exist is that the $M$ value at the top of the trapping layer be less than the $M$ value at the surface. These ducts are not particularly selective as to frequency. Surface ducts can support over-thehorizon propagation at frequencies above $100 \mathrm{MHz}$ and are responsible for most extremely long-range over-the-horizon radar detections and radio communications.

Elevated ducts, as shown in Fig. 1(c), are created by elevated trapping layers similar to those that create surface ducts. However, in this case, the layer is either too high or the $M$ deficit is too small to meet the condition stated above to form a surface duct. The vertical extent of the duct is from the top of the trapping layer down to a height where the $M$ value is equal to the $M$ value at the top of the trapping layer. Elevated ducts also affect propagation for frequencies above approximately $100 \mathrm{MHz}$. For further information on the relationship between refractive effects and propagation see [19].

The parabolic equation (PE) method has been used to model EM propagation in the troposphere for many years. The biggest advantage to using the PE method is that it gives a full-wave (amplitude and phase) solution for the field in the presence of range-dependent environments. Assuming a time dependence of $e^{-i \omega t}$ and that the atmosphere varies in range $x$ and height $z$ the parabolic equation for a flat earth is given by

$$
\frac{\partial^{2} \psi(\mathbf{r})}{\partial z^{2}}+2 i k_{o} \frac{\partial \psi(\mathbf{r})}{\partial x}+k_{o}^{2}\left[n^{2}(\mathbf{r})-1\right] \psi(\mathbf{r})=0
$$

where $k_{o}$ is the free-space wavenumber, $n$ is the index of refraction, and $\psi$ represents a complex scalar component of the electric field. The field from either a horizontal or vertical electric-dipole source satisfies the same parabolic differential equation.

The parabolic equation method was originally developed by Fock [20], but it was not until a number of years later that a practical solution for complicated refractive environments, referred to as the split-step Fourier method, was developed [21]. For range-dependent refractive environments, the complex scalar component of the field at range $x+\Delta x$ can be written as

$$
\begin{aligned}
\psi(x+\Delta x, z)= & \exp ^{i k_{o}(\Delta x / 2)\left(M^{2}(\mathbf{r})-1\right)} \\
& \times F^{-1}\left[\exp ^{-i(\Delta x / 2)\left(k_{z}^{2} / k_{o}\right)} F[\psi(\mathbf{r})]\right]
\end{aligned}
$$

where $F$ and $F^{-1}$ are the Fourier transforms. The transform variable $k_{z}$ is defined by $k_{0} \sin (\theta)$ where $\theta$ is the propagation angle above the horizontal. Additional information on the splitstep PE solution may be found in [22]-[24] and [25] gives a PE model based on a modification to the smooth earth PE to incorporate arbitrary terrain.

\section{Objective Functions for MFP}

The MFP objective function provides a measure of similarity between the observed signal field and the predicted signal field where the observed signal field is the vector-valued array data and the predictions are based on the forward propagation model and environmental parameters. In acoustic MFP, two objective functions have been successfully employed. The linear Bartlett processor [26] is perhaps most popular, though in some situations the nonlinear minimum variance distortionless response (MVDR) processor [27] is preferred. The same options are available for EM-MFP. Whether acoustic or electromagnetic, the MFP objective function is generated as follows: windowed time series from the array are Fourier transformed to form frequency domain data vectors $\mathrm{d}^{l}(\omega)$ where $\omega$ denotes frequency and $l$ the time window. The dimension of the data vectors equals the number of antenna elements. The outer-products of the data vectors are averaged to form the sample covariance matrix

$$
\hat{\mathbf{R}}(\omega)=(1 / L) \sum_{l=1}^{L} \mathrm{~d}^{l}(\omega) \mathbf{d}^{l}(\omega)^{*}
$$

where $*$ denotes conjugate transpose and $L$ is the number of time "snapshots." The normalized Bartlett objective function is then

$$
P_{\mathrm{BT}}(\mathbf{m} ; \omega)=\frac{\mathbf{w}^{*}(\mathbf{m}) \hat{\mathbf{R}}(\omega) \mathbf{w}(\mathbf{m})}{\|\mathbf{w}(\mathbf{m})\|^{2}}
$$

where $\mathbf{w}(\mathbf{m})$ (referred to as the replica vector) is the vector of signal field predictions computed using a forward propagation model and the parameter vector $\mathbf{m}$. Similarly, the minimum variance distortionless response (MVDR) objective function is given by

$$
P_{\mathrm{MV}}(\mathbf{m} ; \omega)=\|\mathbf{w}(\mathbf{m})\|^{2}\left[\mathbf{w}^{*}(\mathbf{m}) \hat{\mathbf{R}}^{-1}(\omega) \mathbf{w}(\mathbf{m})\right]^{-1} .
$$

In acoustic MFP, the MVDR has been shown to outperform the Bartlett processor in terms of sidelobe suppression [28], [29]. Unfortunately, for acoustic MFP it has also been shown that the sensitivity of the MVDR processor to environmental mismatch is significantly greater than that of the Bartlett processor [3]. A number of other objective functions have been investigated for acoustic MFP (see [1] and [2] for a summary of these) but, in general, the Bartlett and MVDR processors have been found to be the most useful.

\section{Global Search Algorithms for MFP}

In early work in acoustic MFP, a 2-D range-depth ambiguity surface was thought to be adequate for source localization 
with a vertical array in a known propagation environment. Such use of a 2-D ambiguity surface was predicated on the implicit assumption that all but two (source location) parameters were known. In that special case, the parameter search was carried out only over the two unknown parameters by plotting the value of the objective function over the range of feasible values for source location. Not surprisingly, in cases where the environmental parameters were, in fact, well known, the ambiguity surface contained a well-defined maximum and the associated source-location estimates were accurate. If the environmental parameters were not well known, then the parameters used to generate the ambiguity surface differed substantially from those of the actual waveguide. In the event of such "mismatch," the ambiguity surface generally contained a number of sidelobes close in amplitude to the maximum and source-location estimation could not be accomplished with any certainty. In that case, there were essentially more than two unknown parameters and, so, as a remedy to mismatch, the maximum of the objective function in an augmented parameter space of dimension greater than two must be found. This requires an efficient global search algorithm.

In Section III, simulation results will be presented for parameter estimation first via a 2-D ambiguity function and then in a space of higher dimension using a global optimization technique referred to as "genetic algorithms." The basic principle of GA is simple: from all possible parameter vectors, an initial population of $q$ members is randomly selected. The "fitness" of each member is computed on the basis of the value of the objective function. Based on the fitness of the members a set of "parents" are selected and, through a randomization, a set of "children" is produced. These children replace the least fit of the original population and the process iterates to develop an overall more fit population. A more detailed description of genetic algorithms and their application to parameter estimation is given in [16].

\section{TROPOSPHERIC SimUlations}

This section presents the results of computer simulations of EM-MFP in the tropospheric setting. As might be expected from the discussion in the earlier sections, the simulations are of three general types. In Section III-A, all environmental parameters are assumed to be known and a range-height ambiguity surface is generated for estimating the unknown source-location parameters. In Section III-B, knowledge of environmental parameters is not assumed and the M-profile parameters are estimated assuming that the source location is known. Finally, in Section III-C the M-profile parameters are estimated simultaneously with the source-location parameters. In Sections III-B and C estimation involves global search/optimization using genetic algorithms as opposed to the exhaustive search performed in Section III-A.

During the GA optimization, all obtained samples of the search space are stored and used to produce a posteriori probability distributions for the parameters. For a system of $p$ parameters, the result is a $p$-dimensional space. This is difficult to display and, therefore, only the marginal probability

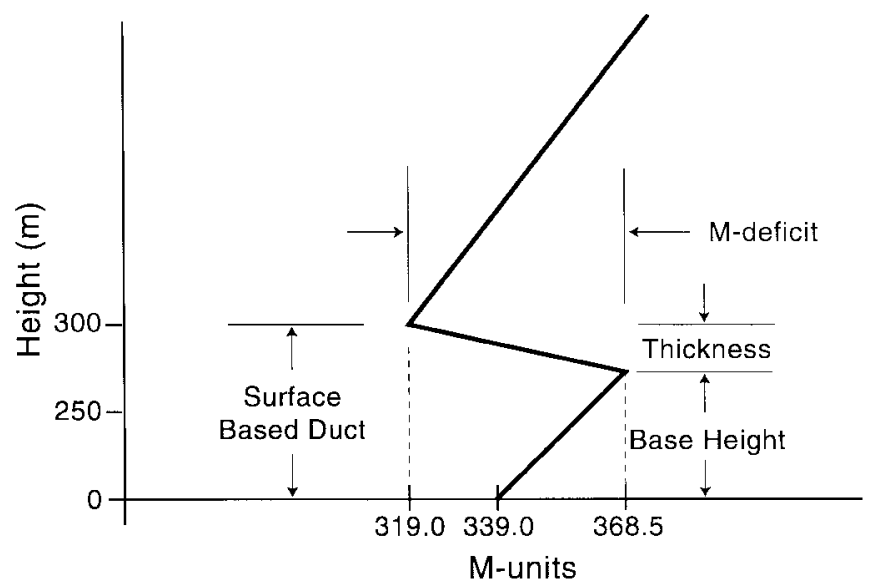

Fig. 2. Modified vertical refractivity for a tri-linear profile.

distributions are shown. The samples are ordered according to their energy and the probability distribution is scaled using a Boltzmann distribution [16]. The a posteriori distributions are useful for evaluating the convergence of the inverse solution and uniqueness of the solution.

In all simulations, the synthetic array data were generated for a scenario with the following general characteristics.

Source Signal: The synthetic signal simulated an omnidirectional point source with horizontal polarization at a frequency of $1 \mathrm{GHz}$. Source ranges were 60,90 , or $120 \mathrm{~km}$ and source height was $50 \mathrm{~m}$.

Receive Antenna: The receive antenna was a vertical array that contained 50 omnidirectional elements. Two configurations were considered: 1) element spacing of $2 \mathrm{~m}$, first element at $2 \mathrm{~m}$ above mean sea level (MSL) and total aperture of 98 $\mathrm{m}$ (or 326.6 wavelengths at $1 \mathrm{GHz}$ ) and 2) element spacing of $1 \mathrm{~m}$, first element at $1 \mathrm{~m}$ above MSL and total aperture of 49 $\mathrm{m}$ (or 163.3 wavelengths at $1 \mathrm{GHz}$ ).

Propagation Environment: The propagation environment used for all synthetic cases was that of a surface duct caused by an elevated trapping layer. Fig. 2 illustrates the tri-linear $M$ profile that characterizes such an environment. The trilinear $\mathrm{M}$ profile used had constant surface and gradient parameters-surface value set at $339 \mathrm{M}$ units and gradient at the surface and above the duct set at the standard modified refractivity gradient of $118 \mathrm{M}$ units $/ \mathrm{km}$. Conversely, the M deficit, trapping layer base height and trapping layer thickness were considered as variable parameters. The nominal values for the variable parameters were set at M-deficit $49 \mathrm{M}$ units, base height $250 \mathrm{~m}$ and layer thickness $50 \mathrm{~m}$, but were considered to have been unknown for the purposes of the simulations presented in Sections III-B and C. This environment is admittedly a simple idealized case; none the less, it represents a reasonable starting point for MFP parameter estimation.

Propagation Code: The terrain parabolic equation model (TPEM) [25] was used for all simulations. TPEM is based on the split-step Fourier transform to solve the parabolic wave equation, which has been shown to be numerically efficient. In fact, TPEM is more capable than necessary for these simula- 


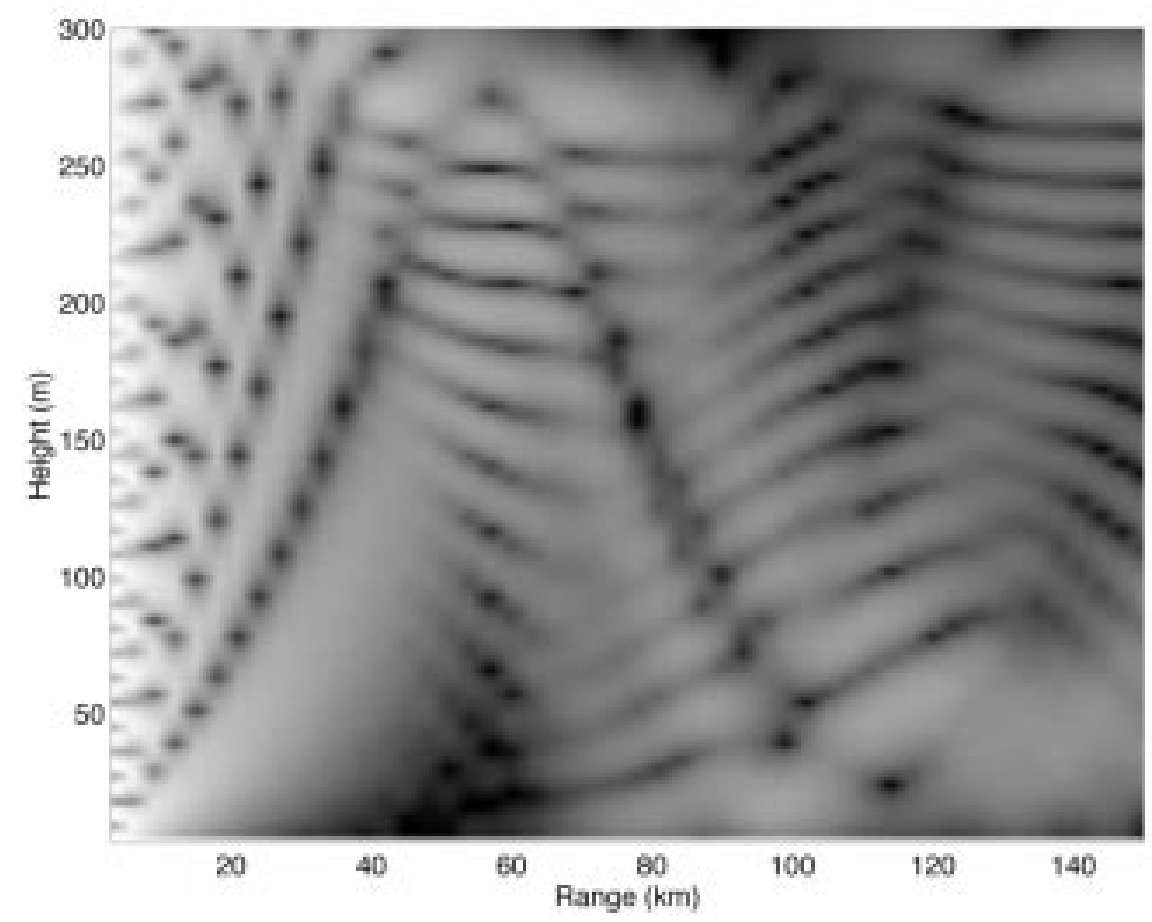

Fig. 3. Coverage diagram showing propagation loss as a function of height and range for the tri-linear refractivity profile (RF emitter at $50 \mathrm{~m}$ ); the frequency is $1 \mathrm{GHz}$. The transmission loss scale is from less than $100 \mathrm{~dB}$ (white) to greater than $150 \mathrm{~dB}$ (black).

tions in that it can predict tropospheric radiowave propagation over land with range-dependent terrain and refractivity.

Objective Function: In all cases, the objective function used was the Bartlett processor of (5). The synthetic signal data at each receive-antenna element was generated using TPEM based on the source signal, receive-element location, and propagation environment. The replica vectors were also generated using the TPEM propagation model. The use of the same propagation model for both the signal and replica vectors was a compromise, but since the major issues to be addressed with MFP are spatial sampling and parameter accuracy, this compromise was justified.

Optimization Parameters: The propagation code and objective function were incorporated into the SAGA code [30]. The GA search parameters were: population size -64 , reproduction size -0.5 , cross-over probability -0.05 , number of iterations for each population-2000, and number of populations-10. Thus for each inversion, 20000 forward modeling runs were performed.

CPU Run Time: For generating the ambiguity surfaces, a grid of 50 range $\times 50$ height samples was used. The calculation of the 2500 forward models required about 8-15 min of CPU time on a DEC Alpha 5/266; the exact value depends on the source to receiver range. The genetic algorithm computations of 20000 forward model runs required about $1-2 \mathrm{~h}$ of CPU time, depending on the source to receiver range.

Fig. 3 illustrates the propagation loss coverage diagram for a range-independent terrain for a source at $50 \mathrm{~m}$ with a frequency of $1 \mathrm{GHz}$ computed using TPEM. From this figure it is seen that the dominant component of the propagation is two beams of energy that are reflected by the upper boundary of the surface duct at a range of about $50 \mathrm{~km}$ and again reflected by the surface at a range of about $100 \mathrm{~km}$. These beams dominate the propagation in the lower $300 \mathrm{~m}$ of the atmosphere.

\section{A. Case A: Source-Location Estimation in a Known Environment}

For this case, only the source-location parameters were estimated, i.e., source range (range between source and receiver) and source height (above MSL). The refractivity M profile was known and the correct values for the refractivity parameters (the nominal values) were used in (5). Since the source-location parameter estimation problem involves only two parameters it was carried out using range-height ambiguity surfaces normalized to a maximum value of $0 \mathrm{~dB}$. The principal result was that for this case, the maximum of the ambiguity surface coincided with the actual source location for all ranges considered.

Fig. 4 gives the ambiguity surfaces computed when the receiver array contained 50 elements spanning the first $98 \mathrm{~m}$ of the atmosphere with the source at a height of $50 \mathrm{~m}$. For Fig. 4(a), the source was located at a range of $60 \mathrm{~km}$ from the receiver array. Significant ambiguity in source height is noted in that there are sidelobes at $15 \mathrm{~m}$ and $80 \mathrm{~m}$ at the source range with the largest sidelobe only $0.5 \mathrm{~dB}$ below the maximum. However, there were basically no ambiguities in range over the search region from 20 to $90 \mathrm{~km}$. Figs. 4(b) and (c) illustrate source-location estimation when the source location was at ranges of 90 and $120 \mathrm{~km}$, respectively. The results for the source located at $90 \mathrm{~km}$ are fairly similar to those obtained when the source was located at $60 \mathrm{~km}$, though in this case the sidelobes were displaced in both range and height (whereas for the $60-\mathrm{km}$ case the sidelobes were only displaced in height) and the largest sidelobe was $0.8 \mathrm{~dB}$ below 


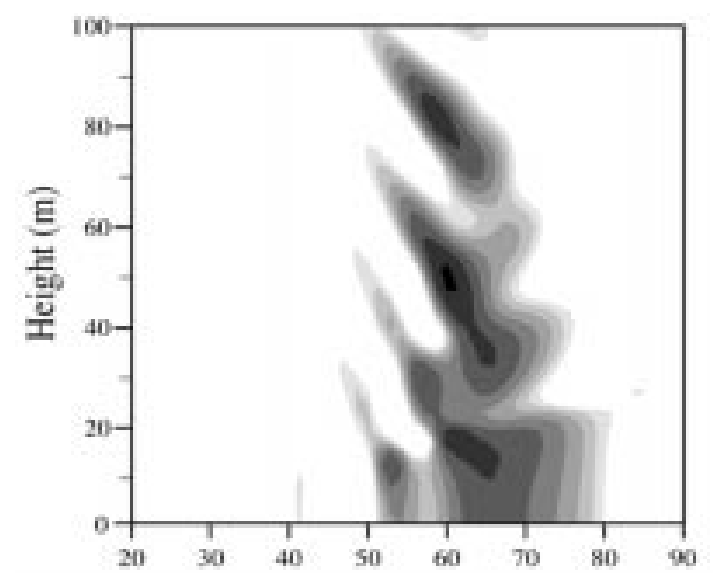

(a)

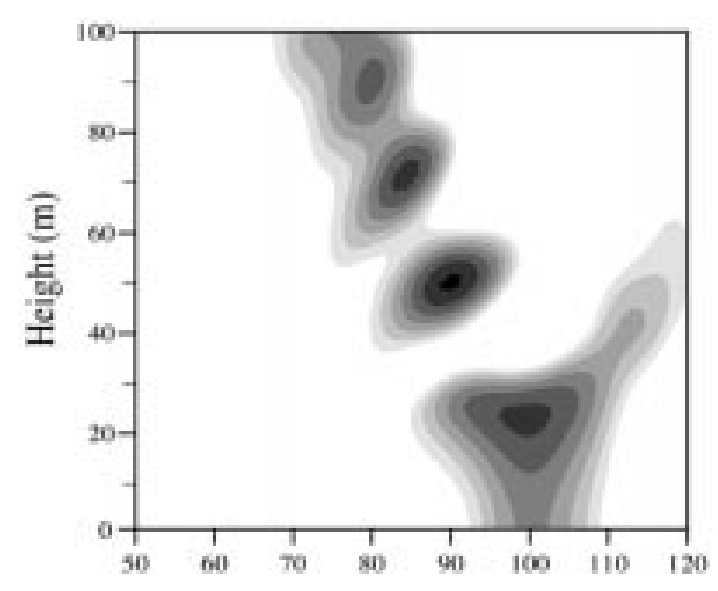

(b)

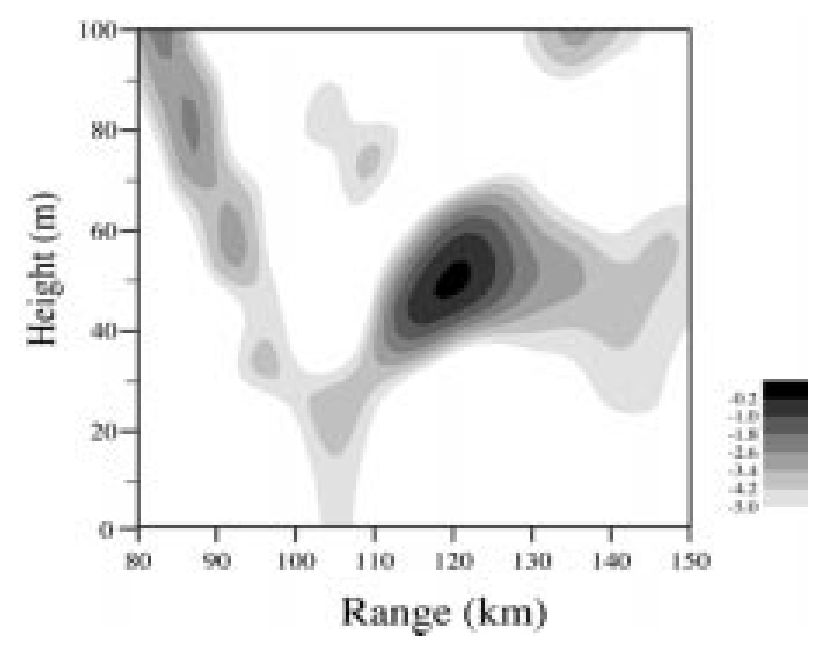

(c)

Fig. 4. Range-height ambiguity surfaces for the known environment case; receive antenna aperture was $98 \mathrm{~m}$, actual source locations were a height of $50 \mathrm{~m}$ and a range of (a) $60 \mathrm{~km}$, (b) $90 \mathrm{~km}$, and (c) $120 \mathrm{~km}$.

the maximum. Fig. 4(c) illustrates the results for a source at $120 \mathrm{~km}$. The results for the source at $120 \mathrm{~km}$ are the best in that the largest sidelobe was $2.6 \mathrm{~dB}$ below the maximum.

As alluded to in the previous section, the relationship between estimation performance and array configuration in MFP is complex. As a point of comparison, a range-height ambiguity surface was computed using an antenna with 50 elements spaced at $1 \mathrm{~m}$, resulting in an aperture of $49 \mathrm{~m}$. Fig. 5 illustrates the result obtained using the 49-m antenna for a source at a range of $90 \mathrm{~km}$ and height of $50 \mathrm{~m}$. Comparing Fig. 5 with the equivalent $98-\mathrm{m}$ aperture result of Fig. 4(b), it is seen that while the source was located correctly in both cases the smaller aperture resulted in an ambiguity surface that was less precise. Overall the resolution was decreased and there were more sidelobes. In addition the sidelobe level increased, the largest sidelobe was only $0.15 \mathrm{~dB}$ below the maximum. Thus, even though both arrays have the same number of elements and are sparse (in that the interelement spacing exceeds a wavelength), performance was degraded with the use of the smaller aperture in spite of the fact that the smaller array more densely samples a high-amplitude region of the signal field (cf. Fig. 3).

\section{B. Case B: Estimation of Environment Parameters, Source Location Known}

The results of MFP simulations for cases in which the three variable M-profile parameters were not known but the source location was known are now discussed. To estimate these parameters via an exhaustive search was not viable (the dimension of the search space for this case was $10^{9}$ ) and so as previously discussed, "genetic algorithms" were used.

Fig. 6 illustrates the result of the GA estimation for the case of a source located at a range of $90 \mathrm{~km}$ and a height of $50 \mathrm{~m}$ using an antenna aperture of $98 \mathrm{~m}$. The a posteriori distributions for each of the three variable M-profile parameters are shown with the actual parameter values for each indicated by a vertical line and the search bounds indicated by the endpoints of each horizontal axis. It is seen that the distributions for the M-deficit and base-height parameters are well defined unimodal peaks located very close to the actual parameter values. The distribution for the layer thickness is not as well defined, but is concentrated around the actual parameter value. Thus, when the source location is known, the threevariable M-profile parameters were accurately estimated and with high confidence for the $\mathrm{M}$ deficit and base height. Fairly similar results were obtained for other source locations.

\section{Case C: Joint Source-Location and Environmental-Parameter Estimation}

For this final case, neither the source location nor the environmental parameters were assumed to be known. The only information provided to the estimation process was that the source range was bounded between 50 and $120 \mathrm{~km}$ and the source height between 0 and $100 \mathrm{~m}$. The actual source location was at a range of $90 \mathrm{~km}$ and a height of $50 \mathrm{~m}$. The bounds for the three refractivity parameters were the same as in Case B. The dimension of the search space for this case was $10^{15}$.

Fig. 7 illustrates the a posteriori distributions for the five estimated parameters using an antenna aperture of $98 \mathrm{~m}$. The source-location distributions are well-defined unimodal distributions with maxima located very close to the actual source-location values. The distributions for the three Mprofile parameters as in Case B, where the source location was known, are well defined and close to the actual values. 

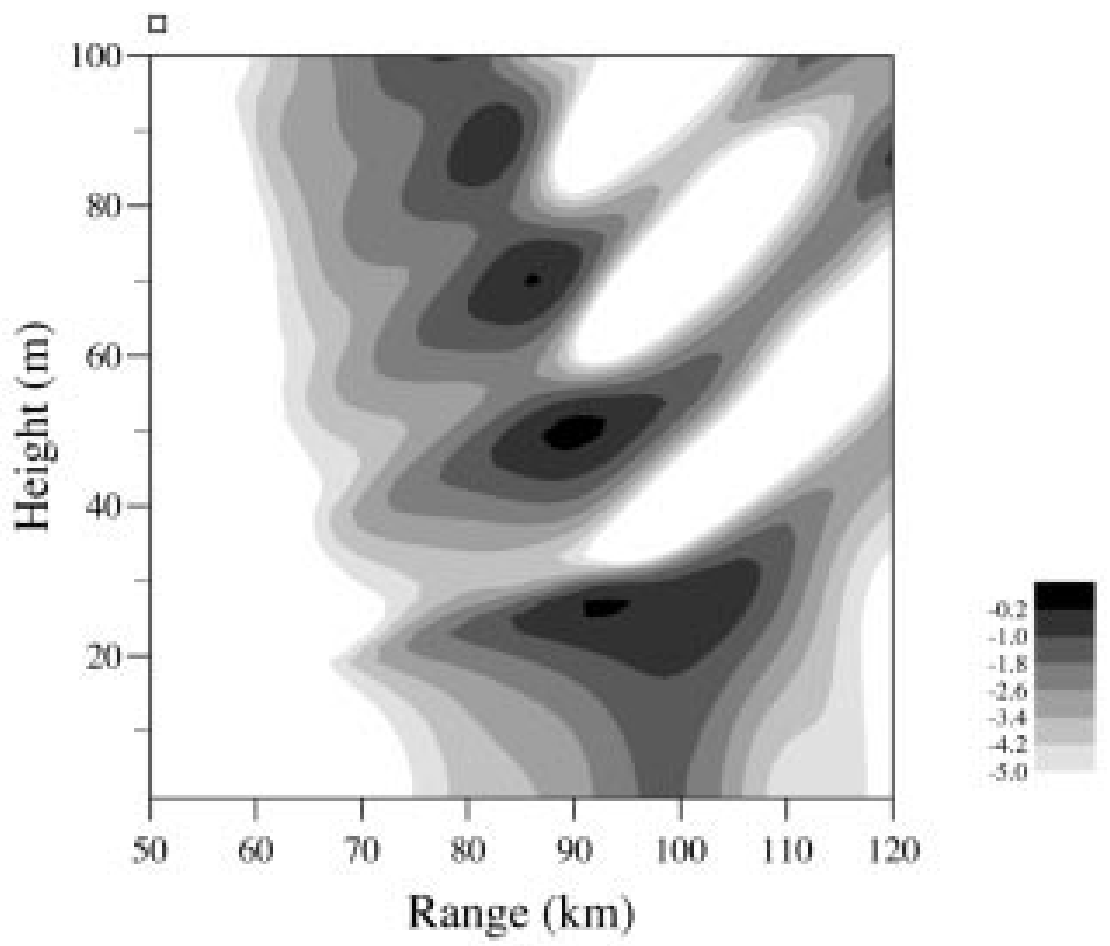

Fig. 5. Range-height ambiguity surface for the known environment case; receive antenna aperture was $49 \mathrm{~m}$, actual source location was a height of $50 \mathrm{~m}$, and range of $90 \mathrm{~km}$.
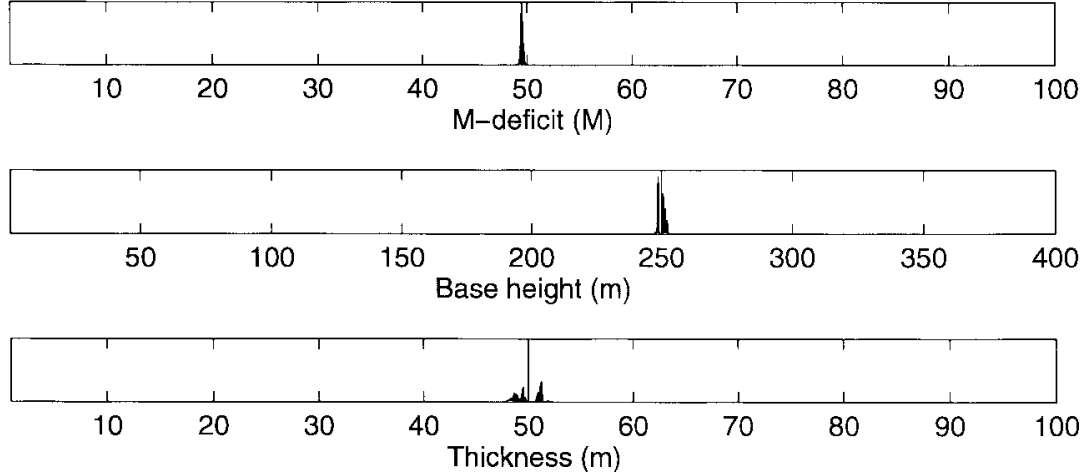

Fig. 6. GA estimated a posteriori distributions for the M-profile parameters, M-deficit, base-height, and layer thickness; antenna aperture was $98 \mathrm{~m}$, source location known.

All five parameter estimates are close to the actual values and confidence is quite high for all of the parameters except the layer thickness.

Fig. 8 illustrates the a posteriori distributions when a $49 \mathrm{~m}$ antenna was used. Comparing this result with that of Fig. 7, it is apparent that performance was seriously degraded when the smaller antenna aperture was used. The estimated sourcelocation parameters and the three M-profile parameters are all in error, especially the three M-profile parameters.

\section{SUMmary AND CONCLUSIONS}

Recent research on array processing for ocean acoustics has included a significant effort to use precise full wave propagation models and efficient parameter search algorithms in order to jointly estimate signal-source location and the environmental parameters that control acoustic propagation. It appears that MFP methods for source localization and environmental parameter estimation have not previously been applied in the electromagnetic domain. In this paper, the extension of MFP to the electromagnetic domain, i.e., EMMFP, as a means for simultaneously localizing an EM source and estimating the environmental parameters associated with the EM propagation was described. Included was a discussion of the basic concepts of MFP and the RF propagation issues associated with tropospheric propagation in the VHF to EHF bands. Solution in the case of unknown source location (range and height for a vertical array) was derived via exhaustive search of the so-called ambiguity surface. For the case of unknown environmental parameters (three refractivity parameters: M-deficit, base-height, and layer thickness) exhaustive 

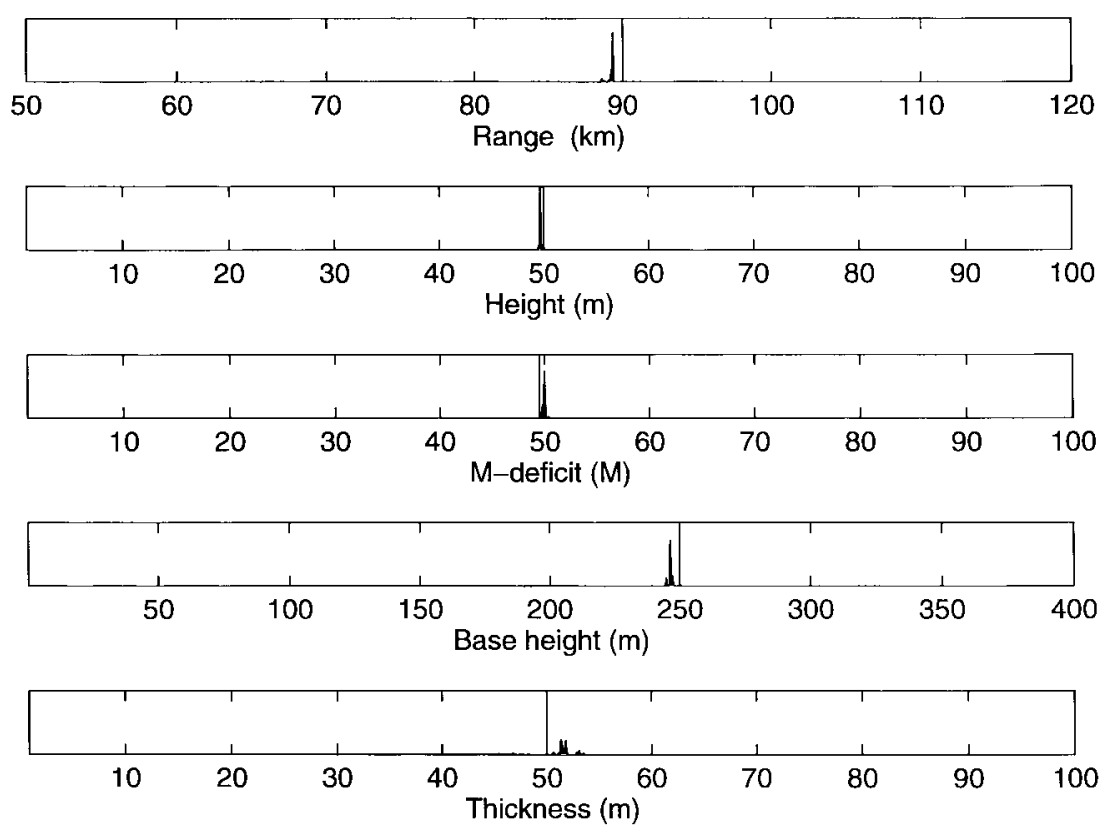

Fig. 7. GA estimated a posteriori distributions for source range, source height, M-deficit, base-height, and layer thickness; antenna aperture was 98 m.
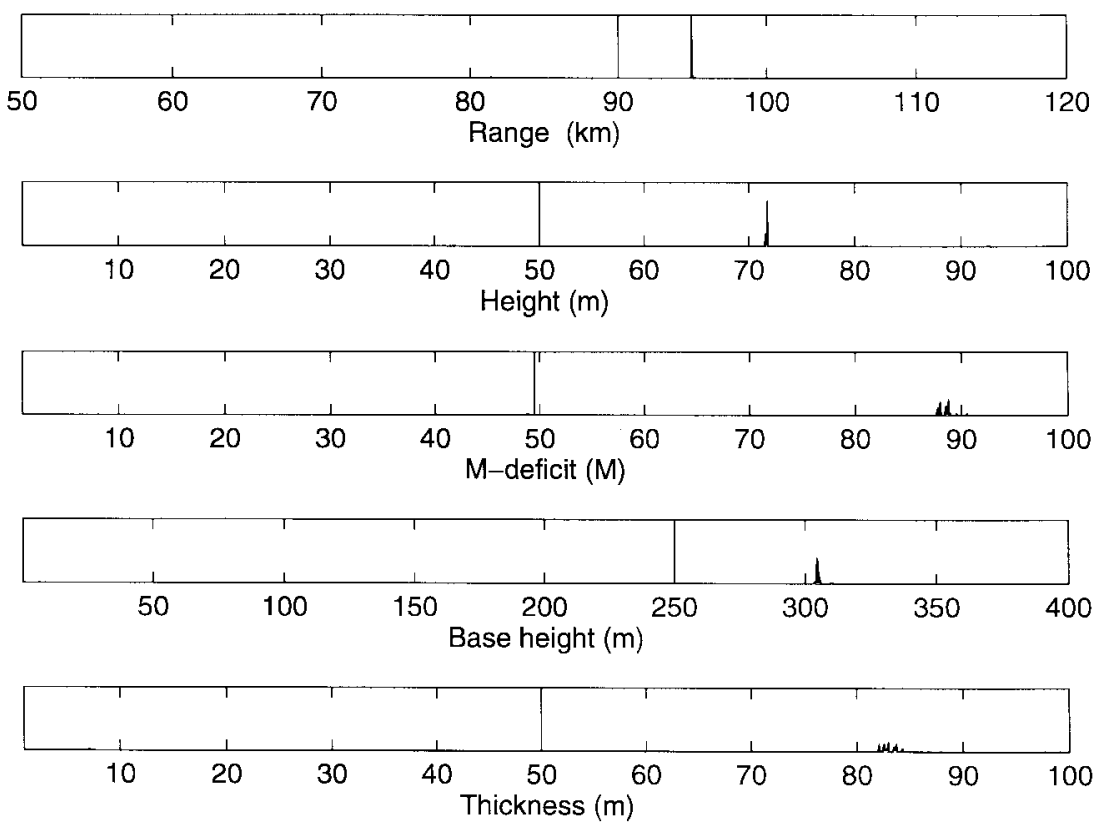

Fig. 8. GA estimated a posteriori distributions for source range, source height, M-deficit, base-height, and layer thickness; antenna aperture was 49 m.

search was not feasible, particularly if source location was also unknown (five parameters total). In this paper, genetic algorithms were used to solve these more difficult cases.

The potential for EM-MFP in the tropospheric setting was illustrated using synthetic data. The simulation results suggest that under suitable conditions, EM-MFP methods can enable EM signal sources to be simultaneously localized and used as sources of opportunity for estimating refractivity. The specific case considered was that of passive localization of a $1-\mathrm{GHz}$ emitter at $50-\mathrm{m}$ height and $60 / 90 / 120-\mathrm{km}$ range in a surface duct environment with a vertical 50-element antenna of length 49/98 m. EM-MFP was carried out using the
TPEM propagation code and Bartlett processor. The results are summarized as follows.

Case A Source location was unknown; three refractivity parameters were known: the ambiguity surface had its maximum at the true source location for all parameter values considered, though for certain values there was ambiguity in source height and/or range, with sidelobes within $0.5 \mathrm{~dB}$ of the maximum.

Case B Source location was known; three refractivity parameters were unknown: a posteriori distributions were computed for the M-deficit, base-height, and 
layer thickness. The distributions for the $\mathrm{M}$ deficit and base height were characterized by well-defined unimodal peaks located very close to the true values. The distribution for the layer thickness was not as well defined but still concentrated around the true value.

Case C Source location was unknown; three refractivity parameters were unknown: a posteriori distributions for source range, source height, $M$ deficit, base height, and layer thickness were computed. For the 98-m aperture the source-location distributions were well-defined and unimodal with peaks located very close to the true source location, and the distributions for the three refractivity parameters were well-defined and close to the actual values. When the 49-m aperture was used, performance was seriously degraded with all five parameter estimates in error, especially the three refractivity parameters.

Even though promising, these results are preliminary and far more needs to be done before EM-MFP can be thought to be of real utility. Of potential interest would be EM-MFP in other EM propagation settings with emitters at other frequencies and with bandwidth using other antenna configurations and in noise. Ultimately, evaluation of EM-MFP using real-field data is required. We note that Krolik [31] has developed EM-MFP-"like" techniques for the ionospheric setting and has demonstrated the possibility of enhanced accuracy in coordinate registration and the potential for height estimation for over-the-horizon (OTH) radar.

\section{REFERENCES}

[1] A. B. Baggeroer, W. A. Kuperman, and P. N. Mikhalevsky, "An overview of matched field methods in ocean acoustics," IEEE J. Oceanic Eng., vol. 18, pp. 401-424, Oct. 1993.

[2] A. Tolstoy, Matched Field Processing for Underwater Acoustics. Singapore: World Scientific, 1993.

[3] D. F. Gingras, "Methods for predicting the sensitivity of matched field processors to mismatch," J. Acoust. Soc. Amer., vol. 86, pp. 1940-1949, 1989.

[4] C. Feuillade, D. R. DelBalzo, and M. M. Rowe, "Environmental mismatch in shallow water matched field processing," J. Acoust. Soc. Amer., vol. 85, pp. 2354-2364, 1989.

[5] A. Tolstoy, "Sensitivity of matched field processing to sound speed profile mismatch for vertical arrays in a deep water Pacific environment," J. Acoust. Soc. Amer., vol. 85, pp. 2394-2404, 1989.

[6] G. B. Smith, H. A. Chandler, and C. Feuillade, "Performance stability of high-resolution matched field processors to sound speed mismatch in a shallow water environment," J. Acoust. Soc. Amer., vol. 93, pp. 2617-2626, 1993

[7] J. C. Preisig, "Adaptive matched field processing in an uncertain propagation environment," IEEE Trans. Signal Processing, vol. 42, pp. 1305-1316, June 1994

[8] J. Krolik, "Matched field minimum variance beamforming in a random ocean channel," J. Acoust. Soc. Amer., vol. 92, pp. 1408-1419, 1992.

[9] D. F. Gingras and N. L. Gerr, "Minimax robust matched field processing," J. Acoust. Soc. Amer., vol. 93, pp. 2798-2809, 1993.

[10] M. D. Collins and W. A. Kuperman, "Focalization: Environmental focusing and source localization," J. Acoust. Soc. Amer., vol. 90, pp. $1410-1422,1991$

[11] D. F. Gingras and P. Gerstoft, "Inversion for geometric and geoacoustic parameters in shallow water: Experimental results," J. Acoust. Soc. Amer., vol. 97, pp. 3589-3598, 1995.

[12] V. R. Noonkester and J. H. Richer, "FM-CW radar sensing of the lower troposphere," Radio Sci., vol. 15, pp. 337-353, 1980.
[13] K. D. Anderson, "Inference of refractivity profiles by satellite-to-ground RF measurements," Radio Sci., vol. 17, pp. 653-663, 1982.

[14] H. V. Hitney, "Remote sensing of the refractivity structure by direct radio measurements at UHF," in AGARD Conf. Proc., Sept. 1992, vol. 502 , pp. $1.1-1.5$

[15] L. T. Rogers, "Likelihood estimation of tropospheric duct parameter from horiziontal propagation measurements," Radio Sci., to be published.

[16] P. Gerstoft, "Inversion of seismoacoustic data using genetic algorithms and a posteriori probability distributions," J. Acoust. Soc. Amer., vol. 95, pp. 770-782, 1994.

[17] A. R. Webster and T. S. Merritt, "Multipath angles-of-arrival on a terrestrial microwave link," IEEE Trans. Commun., vol. 38, pp. 25-30, Jan. 1990.

[18] H. V. Hitney, J. H. Richter, R. A. Pappert, K. D. Anderson, and G. B. Baumgartner, "Tropospheric radio propagation assessment," Proc. IEEE, vol. 73, pp. 265-283, Feb. 1985.

[19] H. V. Hitney, "Refractive effects from VHF to EHF: Part A-Propagation mechanisms," AGARD Lecture Series, vol. LS-196, pp. 4A1-4A13, 1994.

[20] V. A. Fock, "Solutions of the problem of propagation of electromagnetic waves along the earth's surface by method of parabolic equations," $J$. Phys., vol. 10, pp. 13-24, 1946 (USSR).

[21] R. H. Hardin and F. D. Tappert, "Application of the split-step Fourier method to the numerical solution of nonlinear and variable coefficient wave equations," SIAM Rev., vol. 15, pp. 423-434, 1973.

[22] F. D. Tappert, "The parabolic approximation method," in Wave Propagation and Underwater Acoustics, J. B. Keller and J. S. Papadakis, Eds. New York: Springer-Verlag, 1977.

[23] G. D. Dockery, "Modeling electromagnetic wave propagation in the troposphere using the parabolic equation," IEEE Trans. Antennas Propagat., vol. 36, pp. 1464-1470, Oct. 1988

[24] A. E. Barrios, "Parabolic equation modeling in horizontally inhomogeneous environments," IEEE Trans. Antennas Propagat., vol. 40, pp. 791-797, July 1992

[25] _ "A terrain parabolic equation model for propagation in the troposphere," IEEE Trans. Antennas Propagat., vol. 42, pp. 90-98, Jan. 1994.

[26] H. P. Bucker, "Use of calculated sound fields and matched field detection to locate sound sources in shallow water," J. Acoust. Soc. Amer., vol. 59, pp. 368-373, 1976.

[27] J. Capon, "High resolution frequency wavenumber spectrum analysis," Proc. IEEE, vol. 57, pp. 1408-1418, Aug. 1969.

[28] R. G. Fizell, "Application of high-resolution processing to range and depth estimation using ambiguity function methods," J. Acoust Soc. Amer., vol. 82, pp. 606-613, 1987.

[29] A. B. Baggeroer, W. A. Kuperman, and H. Schmidt, "Matched field processing: Source localization in correlated noise as an optimum parameter estimation problem," J. Acoust. Soc. Amer., vol. 83, pp. 571-587, 1988

[30] P. Gerstoft, SAGA Users Guide 2.0-An Inversion Software Package, SACLANT Undersea Res. Ctr., La Spezia, Italy, SM-333, 1997.

[31] J. L. Krolik and R. H. Anderson, "Maximum likelihood coordinate registration for over-the-horizon radar," IEEE Trans. Signal Processing, vol. 45, pp. 945-959, Apr. 1997.

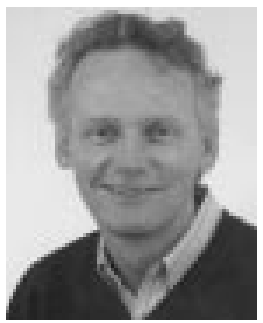

Donald F. Gingras (S'68-M'68) was born June 4, 1945 in San Diego, CA. He received the B.S. degree from San Diego State University, San Diego, CA, in 1968, the M.S. degree from Northeastern University, Boston, MA, in 1971, and the Ph.D. degree from the University of California, San Diego, in 1986, all in electrical engineering.

From 1968 to 1971, he was a Sonar Design Engineer with Raytheon at the Submarine Signal Division, Portsmouth, RI. From 1971 to 1990 he worked in acoustic signal processing at the Naval Command Control and Ocean Surveillance Center in San Diego, CA. From 1990 to 1995 he lead the Signal Processing Group at the SACLANT Undersea Research Centre, La Spezia, Italy. In 1996 he returned to the Naval Command Control and Ocean Surveillance Center in San Diego, CA. His current research interests include matched-field processing, propagation modeling, wireless communications, and code-division multiple access systems. 


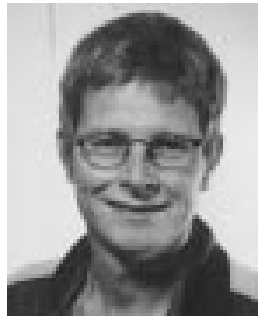

Peter Gerstoft received the M.Sc. degree from both the Technical University of Denmark, Lyngby, in 1983, and from the University of Western Ontario, London, Canada, in 1984, and the Ph.D. degree from the Technical University of Denmark, in 1986.

From 1987 to 1992, he was employed at Ødegaard and Danneskiold-Samsøe, Copenhagen, Denmark, working on forward modeling and inversion for seismic exploration. From 1989 to 1990 he was Visiting Scientist at Massachusetts Institute of Technology, Cambridge, and at Woods Hole Oceanographic Institute, Woods Hole, MA. From 1992 to 1997 he was a Senior Scientist at SACLANT Undersea Research Centre, La Spezia, Italy, where he has developed the SAGA inversion code. Since 1997 he has been with the Marine Physics Laboratory at the University of California, San Diego, CA. His research interests include global optimization, modeling and inversion of acoustic, elastic, and electromagnetic signals.

Dr. Gerstoft is a member of Society of Exploration Geophysics and Acoustical Society of America.

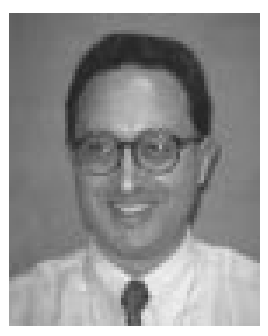

Neil L. Gerr (M'84) was born February 12, 1954, in New York, NY. He received the B.S. degree in mathematics from the Massachusetts Institute of Technology, Cambridge, MA, in 1976, and the M.S and $\mathrm{Ph} . \mathrm{D}$. degrees in statistics from the University of North Carolina at Chapel Hill, in 1980 and 1982, respectively.

From 1982 to 1984, he was with Daniel H. Wagner Associates, Inc., Vienna, VA, where he worked on multitarget tracking and naval exercise reconstruction. In 1984 he joined the Office of Naval Research (ONR), McLean, VA, where he remains today. As Scientific Officer for Signal Analysis from 1984 to 1988 he was responsible for managing a program of basic and applied research in stochastic process theory and time series analysis with applications in antisubmarine warfare. From 1988 to 1993 he served as Director of the Mathematical Sciences Division with overall responsibility for research programs in applied analysis, discrete mathematics, numerical analysis, operations research, probability and statistics, and signal analysis. In 1994 he was named Director of the newly formed Surveillance, Communications, and Electronic Combat Division. In this capacity he provides leadership and oversight for research and development programs in radar and electro-optics/infrared (EO/IR) sensors; signal, array and image processing; radio communications and wireless networks; threat warning, self protection, and electronic warfare mission support; and navigation. He has published journal papers in the areas of statistical communication theory, spectral analysis chaotic time series, signal processing, and array processing.

Dr. Gerr is a member of the Society for Industrial and Applied Mathematics and the Acoustical Society of America. 